\title{
Erstdiagnose eines systemischen Lupus erythematodes in der 15. SSW mit Gemini
}

\section{First Time Diagnosis of Systemic Lupus erythematosus in the 15th Week of Pregnancy with Twins}

Autoren

Institut

\section{B. Stevens, P. von den Driesch}

Klinik für Dermatologie und Allergologie, Zentrum für Hautkrankheiten, Klinikum Stuttgart

\section{Bibliografie}

DOI 10.1055/s-2007-966522

Akt Dermatol 2007; 33:

228-231 @ Georg Thieme

Verlag KG Stuttgart · New York ISSN 0340-2541

Korrespondenzadresse

Dr. med. Birthe Stevens Klinik für Dermatologie und Allergologie, Zentrum für Hautkrankheiten, Klinikum Stuttgart Prießnitzweg 24

70327 Stuttgart

b.stevens@kbc-intern.de

\section{Zusammenfassung \\ $\nabla$}

Lange Zeit hat man Patientinnen mit systemischem Lupus erythematodes von einer Schwangerschaft abgeraten. Erfreulicherweise ist in den letzten Jahren eine Schwangerschaft bei Frauen mit SLE zur Normalität geworden. Dennoch ist hier eine große Anzahl von Komplikationen möglich, vor allem bei einer zusätzlichen Nierenbeteiligung oder dem Vorliegen eines Antiphospho-

\section{Einleitung \\ $\nabla$}

Beim systemischen Lupus erythematodes handelt es sich um eine schubweise verlaufende, chronisch-entzündliche Autoimmunkrankheit des Bindegewebes mit Hautaffektionen und der Möglichkeit der Beteiligung zahlreicher Organsysteme. Charakteristisch ist der Nachweis von Autoantikörpern gegen Zellkernbestandteile. Die Inzidenz der Erkrankung liegt in Deutschland bei etwa 20-30 Erkrankten pro 100000 Einwohner [3]. Da Frauen 8-10-mal häufiger betroffen sind als Männer und die Erstmanifestation der Erkrankung häufig im gebärfähigen Alter liegt, stellt sich die Frage der Risiken durch die Erkrankung im Falle einer Schwangerschaft für Mutter und Kind. Hier wurde noch bis vor wenigen Jahren von einer Schwangerschaft grundsätzlich abgeraten - aus Furcht vor einer Aktivierung der Erkrankung und aufgrund eines mit der Krankheit verbundenen Risikos für die Kinder. Die kindliche Sterblichkeit lag durchschnittlich bei 30\% [3]. Mittlerweile sind deutlich mehr Erfahrungen mit dem klinischen Management dieser Risikoschwangerschaften vorhanden, die oben genannten Hauptrisiken können benannt und konkrete Empfehlungen zum Umgang mit diesen gegeben werden. Es herrscht jedoch noch kein Konsens und es fehlen nach wie vor allgemeine Leitlinien. Unumstritten jedoch ist die Notwendigkeit einer
lipid-Antikörper-Syndroms. Reaktivierung des SLE, Thrombose, Fehlgeburt, Neugeborenenlupus, Schwangerschafts-induzierte Hypertonie, pulmonale Hypertonie und Arzneimitteltoxizität zu beachten. Wir stellen den Fall einer mit Zwillingen schwangeren Patientin vor, bei der wir in der 15. SSW die Erstdiagnose eines systemischen LE stellten, und nehmen dies zum Anlass, über die aktuellen Empfehlungen bezüglich solcher Risikoschwangerschaften zu informieren.

interdisziplinären, sehr engmaschigen Betreuung dieser Patientengruppe während der Schwangerschaft und im Wochenbett (Auftreten eines SLESchubes bei ca. $65 \%$ der Patientinnen besonders im 2. Trimenon und post partum). Wir berichten über den seltenen Fall einer Zwillingsschwangerschaft, bei der ein SLE erstmals auftrat.

\section{Fallbericht \\ $\nabla$}

Anamnese

Ambulante Vorstellung einer 38-jährigen Patientin in deutlich reduziertem Allgemeinzustand in der 15. SSW ihrer dritten Schwangerschaft. Komplikationsloser Verlauf der beiden vorausgegangenen Schwangerschaften. Aktuell seit 3 Tagen Auftreten multipler brennender und juckender „roter Flecken“ mit Beginn an beiden Wangen, im Verlauf Übergang auf Decolleté und beide Arme. Seit Beginn der Schwangerschaft fühle sich die Patientin unnatürlich müde und geschwächt, antriebsarm und lustlos, was ihr von ihren vorherigen Schwangerschaften, in denen sie sich immer wohl und voller Tatendrang gefühlt habe, nicht bekannt sei. Seit Monaten täglich wässriger Durchfall, aktuell stressbeladene häusliche Situation, bekannter Herpes labialis recidivans, derzeit abgeheilt. Z.n. ausgeprägtem grippalen Infekt vor 8 Wochen, keine Gelenk- oder Muskelbeschwer- 


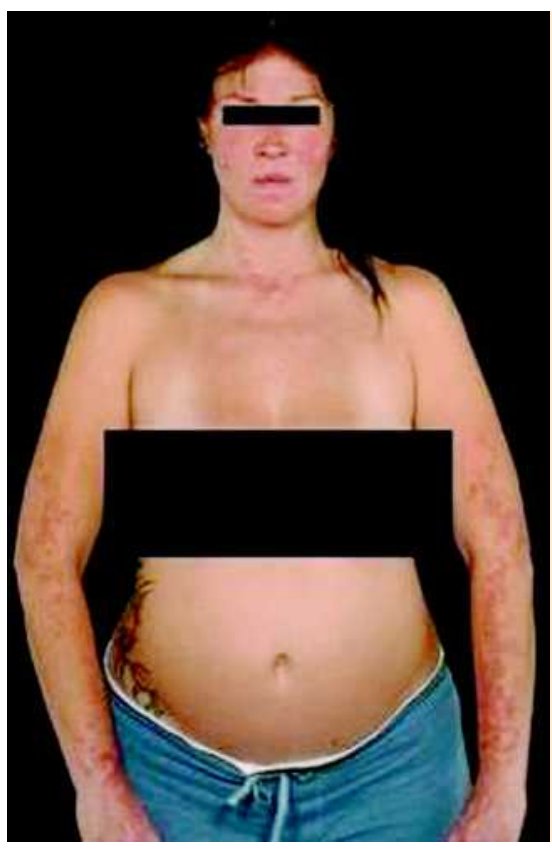

Abb. 1 Teils erythematöse, teils leicht urtikariell imponierende konfluierende Erytheme mit Betonung der lichtexponierten Areale (Übersicht).

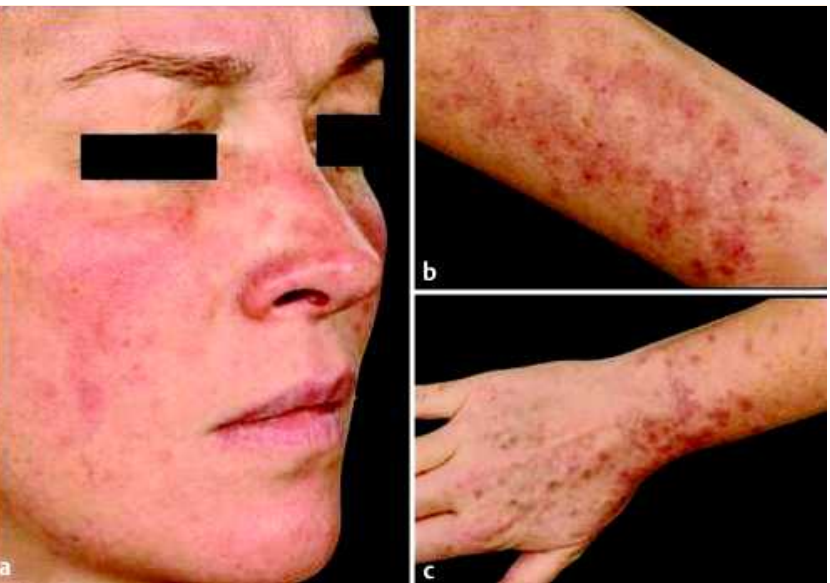

Abb. 2 Typisches Schmetterlingserythem mit Aussparung der Nasolabialfalten (a), Erosionen nach zentraler Bläschenbildung bei makulopapulösem konfluenten Erythem (b), Betonung der Streckseiten und Mitbefall der Hände (b und c).

den, keine Nervenentzündungen, keine Krampfanfälle, jedoch ausgeprägte rezidivierende dumpf dröhnende Kopfschmerzen und wiederkehrende Ulzerationen am Gaumen in wechselnder Lokalisation, auch wenn der bekannte Herpes gerade nicht aktiv sei. Zudem seit Jahren bekannte sehr lichtempfindliche Haut, hier sei es im Sommer 2005 sogar zu nässenden Hautveränderungen an Gesicht, Oberarmen und Decolleté gekommen, die zunächst als „Sonnenallergie“, später als Lichtdermatose eingestuft worden seien. Zusammen mit diesen Hauterscheinungen sei es häufiger zu regelrechten allgemeinen Erschöpfungszuständen gekommen. Es erfolgte die stationäre Aufnahme der Patientin bei uns unter dem dringenden klinischen Verdacht auf einen Lupus erythematodes zur weiteren Diagnostik, Durchuntersuchung und Therapieeinleitung.

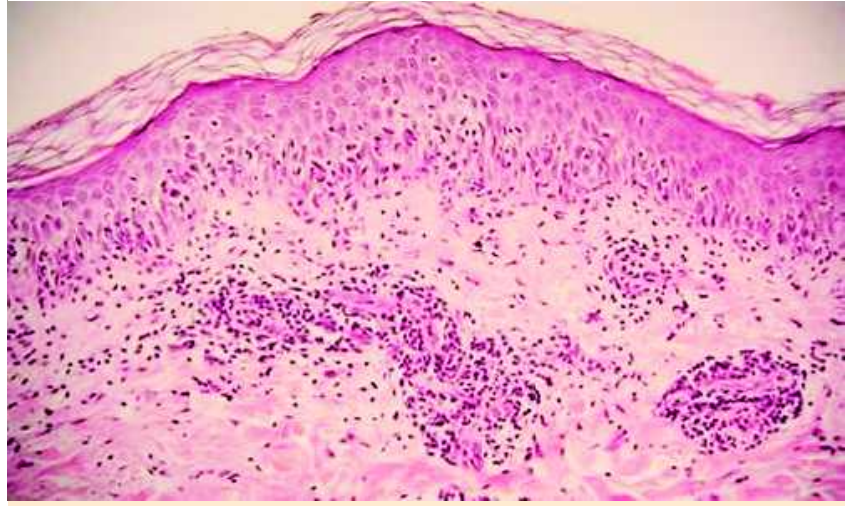

Abb. 3 Mäßige Spongiose, Basalzelldegeneration, ausgeprägte Interface-Dermatitis. Im oberen Korium Pigmentinkontinenz sowie ein relativ ausgeprägtes, deutlich perivaskulär akzentuiertes, vorwiegend lymphozytäres Infiltrat. Im tiefen Korium und der Subkutis allenfalls diskrete entzündliche Infiltrate um die korialen Gefäße herum zu finden. Somit gut vereinbar mit einem recht akuten Lupus erythematodes.

\section{Dermatologischer Aufnahmebefund}

Teils papulöse, teils leicht urtikariell imponierende konfluierende Erytheme, teils mit zentraler Blasenbildung an beiden Armen und Decolleté, Halsdreieck ausgespart, im Gesicht Schmetterlingserythem mit Aussparung der Nasolabialfalten ( Abb. 1 u. 2). Nebenbefundlich Varikosis des linken Beines. Mundschleimhaut, restliches Integument einschließlich Nagelfalze unauffällig.

\section{Histologie}

Histologie-Nr. 8287/06: Innerhalb der Epidermis mäßige Spongiose sowie Degeneration der Basalzellen. Ausgeprägte Interface-Dermatitis mit Lymphozyten, die auch in höheren Epidermislagen zu finden sind. Im oberen Korium zeigt sich eine Pigmentinkontinenz sowie ein relativ ausgeprägtes, deutlich perivaskulär akzentuiertes, vorwiegend lymphozytäres Infiltrat. Das tiefe Korium sowie die Subkutis stellen sich soweit unauffällig dar. Dort sind allenfalls diskrete entzündliche Infiltrate um die korialen Gefäße herum zu finden ( $\bullet$ Abb. 3).

Histologisch gut vereinbar mit einem Lupus erythematodes.

\section{Direkte Immunfluoreszenz}

Diskrete granuläre Immunfluoreszenz von IgM: vereinbar mit LE, kein Anhalt für Pemphigoid gravidarum.

\section{Labor bei Aufnahme}

Pathologisch verändert

ANA-Screening ( $\operatorname{IgG}) 1: 160$, Fluoreszenzmuster fein gesprenkelt, CRP 2,1 mg/dl (bei Entlassung 0,4 mg/dl), Kreatinin 0,4 mg/ dl (0,5-0,9), Leukozyten 10,41 T/ul (4-10), Kreatinin im Urin 2,1 g/die (<1,5); g Protein/g Kreatinin 0,2; Eiweiß im Urin quant. $17,5 \mathrm{mg} / \mathrm{dl}$; Eiweiß im Urin $420 \mathrm{mg} / \mathrm{die}$, BZTP max. Werte bis $126 \mathrm{mg} / \mathrm{dl}$; Elektrophorese: Albumin 48,8\% (60,3-71,4); alpha 1-Glob. 3,7\% (1,4-2,9); alpha 2-Glob. 17,5\% (7,2-11,3), betaGlob. $15,1 \%(8,1-12,7)$.

\section{Im Normbereich}

Restliches Differenzialblutbild, Glukose, Elektrolyte, Gerinnung, gamma-Globulin-Elektrophorese, Rheumafaktor, epidermale und Stachelzelldesmosomen-Autoantikörper, ds-DNA-AK, Ena- 
Profil (insbesondere Ro/SS-A und La/SS-B), Antiphospholipidund Anticardiolipin-Antikörper, Kreatinin-Clearance.

\section{Stuhluntersuchung}

Kein Wachstum von Salmonellen, Shigellen, Campylobacter oder Yersinien.

\section{Apparative Untersuchungen}

EKG, Echokardiografie, MRT-Schädel nativ mit Duplex, neurologisches Konsil ohne Anhalt für Systembeteiligung; nebenbefundlich im neurologischen Konsil V.a. Spannungskopfschmerz.

\section{Gynäkologisches Konsil}

Gemini 15. SSW intakt; Aufnahme der Patientin in einen regelmäßigen Kontrollrhythmus in der Schwangerenambulanz der Klinik.

\section{ARA-Kriterien}

Mit Schmetterlingserythem, deutlich erhöhter Lichtempfindlichkeit der Haut, rezidivierenden schmerzlosen Ulzerationen im Mundbereich, erhöhten antinukleären Antikörpern waren 4 von 11 Kriterien positiv.

\section{Therapie und Verlauf}

Unter der klinischen Diagnose eines drohenden SLE wurde bei deutlich reduziertem Allgemeinzustand der Patientin eine systemische Therapie mit Methylprednisolon initial $60 \mathrm{mg} /$ die unter Magenschutz mit Maaloxan und Osteoporoseprophylaxe (Calcium und Vitamin D3) eingeleitet. Diese wurde zügig in 5 Tagen reduziert auf $20 \mathrm{mg} /$ die. Es zeigte sich bereits bei Entlassung eine Verbesserung des Allgemeinbefindens sowie eine Abnahme der Aktivität der beschriebenen Hautveränderungen im Sinne eines Abblassens. Die Patientin wurde in eine engmaschige Kontrolle durch unsere Ambulanz und die Schwangerenambulanz der Frauenklinik des Klinikums Stuttgart eingebunden, wo aufgrund einer erfahrenen Neonatologie die Entbindung geplant ist. In unserer Ambulanz konnte bei abklingender Symptomatik die Methylprednisolon-Dosis weiter reduziert werden. Gemeinsame Entscheidung mit den betreuenden Gynäkologen für eine zusätzliche Dauermedikation der Patientin mit Acetylsalizylsäure während der Schwangerschaft zur Vermeidung thromboembolischer Komplikationen. Aufgrund der o.b. leichten Proteinurie erfolgte eine Vorstellung der Patientin beim Nephrologen, der einen Nierenbefall bei SLE ausschloss.

\section{Diskussion}

$\nabla$

Immunologisch betrachtet ist die Schwangerschaft eine Phase der Anpassung der mütterlichen Immunität zur Vermeidung der Abstoßung des Fetalgewebes. In der Schwangerschaft wechselt die Immunantwort von einem Th1- zu einem Th2-Profil mit einer erhöhten Expression der Interleukine 4 und 10, die mit der Pathophysiologie des SLE in Verbindung gebracht wurden [1]. Weiter unterstützt das Auftreten von SLE-Schüben in der Schwangerschaft und im Wochenbett die Hypothese, dass den Östrogenen als Kofaktor in der Pathogenese der Erkrankung eine wichtige Rolle zukommt [6]. Es herrscht weitgehend Konsens in der Literatur, dass Lupusmanifestationen in der Schwangerschaft nicht gravierender sind, die meisten Schübe betreffen Haut und Gelenke, schwerwiegendere Verläufe kommen jedoch vor [7]. Frühgeburtlichkeit und Fetalmortalität sind mit LE-Akti- vität und aktiver Nephritis in Verbindung gebracht worden [7], das Risiko eines kongenitalen Herzblocks beim Kind mit dem Vorkommen von Ro/SS-A und La/SS-B Antikörpern, die während des aktiven IgG-Transportes zwischen der 16. und 30. SSW über die Plazenta in den fetalen Kreislauf gelangen $[1,6]$. Liegt zudem ein Antiphospholipidsyndrom (APS) vor, unterliegen die Schwangeren einem erhöhten Risiko einer Fehlgeburt oder arteriellen wie venösen thromboembolischen Ereignissen. Hier haben Untersuchungen der Plazenten von Frauen mit APS für das Vorliegen einer Plazentainsuffizienz Plazentathrombosen ergeben [1]. Mit einer früheren Lupus-Nephritis sowie auch mit einem APS werden weiter ein erhöhtes Risiko für eine Präeklampsie (schwangerschaftsinduzierte Hypertonie plus Proteinurie von mindestens $300 \mathrm{mg} / \mathrm{d}$ ) in Verbindung gebracht [8].

Aus diesen Risiken leiten sich die nachfolgenden Empfehlungen $\mathrm{ab}$ :

Der Lupus erythematodes sollte mindestens 6 Monate vor Beginn einer Schwangerschaft inaktiv gewesen sein. Kontraindikationen für eine Schwangerschaft bestehen in der pulmonalen Hypertonie (durch SS und Geburt erhöht sich die Herzbelastung erheblich, Schwangerschafts-induzierte Mortalität, v.a. postpartal: bis zu 50\%!), einem aktiven Schub des SLE zum Zeitpunkt der Konzeption und einer aktiven Lupusnephritis. Wird eine Schwangerschaft geplant, sollte eine etwaige vorbestehende Therapie des LE auf ihre Notwendigkeit hin überprüft, wenn möglich verringert oder abgesetzt werden, natürlich unter dann engmaschiger Kontrolle des Verlaufs. Nach Absetzen sollte vor der Konzeption möglichst drei Monate abgewartet werden, um auf eine etwaige Reaktivierung der Erkrankung reagieren zu können [3]. Tritt eine Schwangerschaft unter laufender Therapie ein, sollte die Behandlung der Mutter möglichst unverändert bleiben [1]. In der Literatur zu diesem Thema wird die Gabe von Hydroxychloroquin überwiegend nicht als schädlich für den Fetus eingestuft [1,3]. Prednison und Methylprednisolon werden ebenfalls generell als unbedenklich eingestuft, hohe Kortisondosen ab der 20. SSW erhöhen jedoch das Risiko einer Präeklampsie und eines vorzeitigen Blasensprungs, Dosierungen $>15-20 \mathrm{mg} / \mathrm{d}$ sollten nicht über längere Zeit gegeben werden. Bei hoher Aktivität kann Methylprednisolon als Bolus mit bis zu 500 mg verabreicht werden [1]. Auch Azathioprin und Cyclosporin können durchaus in der Schwangerschaft gegeben werden angesichts der möglichen schweren Konsequenzen eines Krankheitsschubs für Mutter und Kind ist es nicht erforderlich, bei Empfängnis unter dieser Medikation dieselbe abzusetzen [1,3]. Cyclophosphamid, Mycophenolatmofetil und Methotrexat sind aufgrund des hohen Abort- und Fehlbildungsrisikos kontraindiziert $[1,3]$.

Bei dem Vorliegen positiver Ro/SS-A und La/SS-B-Autoantikörper werden zwischen der 16. und 34. SSW regelmäßige serielle fetale Echokardiogramme empfohlen. Wird ein unvollständiger fetaler Herzblock festgestellt, ist eine Therapie mit den fluorierten Steroiden Dexamethason oder Betamethason empfohlen, welche die Plazentaschranke passieren, da die Chance auf vollständige oder partielle Reversibilität besteht [9]. Auch Kinder mit Myokarditis, Hydrops oder Aszites sind zu behandeln [1]. Liegt bereits ein etablierter vollständiger Herzblock vor, ist die Effektivität einer Steroidgabe umstritten [1]. Bei der Wahl zwischen der Gabe von Dexamethason und Betamethason verschiebt sich aufgrund neuer Studien über einen Zusammenhang zwischen neurologischen Komplikationen beim Neugeborenen und der Langzeitgabe von Dexamethason die Präferenz in Richtung Betamethason [1]. 
Zur Verringerung des Risikos einer Präeklampsie wird ein engmaschiges Monitoring (RR, Proteinurie, Doppleruntersuchungen der Uterusarterien) von Schwangeren mit einem erhöhten Risiko (APS, Nierenbeteiligung bei SLE) empfohlen sowie weiter die gering dosierte Gabe von Acetylsalizylsäure [1].

Bei unserer Patientin kam es im 2. Trimenon einer Zwillingsschwangerschaft zu einem ersten deutlichen LE-Schub, der die Diagnosestellung zuließ. Vorausgegangen waren ein schwerer grippaler Infekt und eine sehr stressbeladene Zeit aufgrund der aktuellen häuslichen Situation. Wir stellten klinisch die Diagnose der generalisierten Form eines akut kutanen Lupus erythematodes (ACLE) nach der Düsseldorfer Klassifikation des kutanen Lupus erythematodes [10-12]. Diese Einordnung erfolgte aufgrund der akuten makulopapulösen exanthematischen Eruption der Hautveränderungen, ihrer pruritischen Komponente sowie der Prädilektion der UV-exponierten Areale und des Gesichtes. Dieser Subtyp des kutanen LE ist für 20-60\% großer Kohorten von SLE-Patienten beschrieben und geht dem Erstauftreten eines systemischen Lupus erythematodes oft um Wochen oder Monate voraus [10]. Als Auslösefaktoren für den ACLE gelten UVExposition, Infektionen (v.a. subtile Virusinfektionen) und verschiedene Medikamente wie z. B. Hydralazin, Isoniazid und Procainamid. Zudem ist eine Assoziation mit HLA-DR2 und -DR3 beschrieben [10].

Aufgrund des häufigen Vorläufercharakters des ACLE für den SLE erfolgte eine umfangreiche Durchuntersuchung der Patientin zur Abklärung einer gegebenenfalls gravierenden Systembeteiligung, die besonders in der Schwangerschaft ein großes Risiko für Mutter und Kind darstellt. Erfreulicherweise konnten wir mit Ausnahme des deutlich reduzierten Allgemeinzustandes der Patientin, erhöhten Werten der antinukleären Antikörper und einer noch unter der Grenze der Anwendbarkeit für die ARA-Kriterien liegenden Proteinurie keinerlei weitere Organbeteiligung feststellen. Im Besonderen waren die Ro/SS-A und La/SS-B-Antikörper nicht erhöht. Somit lag kein erhöhtes Risiko für das Auftreten eines kongenitalen Herzblocks bei den Gemini vor. Mit vier positiven ARA-Kriterien bestand jedoch ein initialer systemischer Lupus erythematodes. Hier entschlossen wir uns bei zunehmender Verschlechterung des Allgemeinzustandes der Patientin und deutlich fortschreitenden Hautveränderungen zu einer kurzfristigen mittelhoch dosierten systemischen Kortikoidgabe eines nicht plazentagängigen Steroids (Methylprednisolon) mit entsprechender Begleitmedikation (s.o.), deren Reduktion bei Ansprechen der Patientin zügig erfolgte. Weiter Gabe von niedrig dosiertem ASS bei durch den SLE auch ohne APS bestehendem erhöhten Risiko für Thrombosen und Embolien, welches durch die Gemini-Schwangerschaft noch verstärkt ist. Hier natürlich auch Empfehlung des Tragens von Kompressionsstrümpfen bei Varikosis des linken Beines. Zudem Einbindung der Patientin in die gynäkologische SchwangerschaftsSprechstunde sowie Einbeziehung eines Nephrologen. Ohne ein interdisziplinäres engmaschiges Management ist bei diesem komplexen Krankheitsbild nicht auszukommen. Schwangere mit SLE sollten möglichst in Studien eingebunden werden, um das Management der möglichen Komplikationen auch in $\mathrm{Zu}$ kunft weiter zu verbessern.

\section{Abstract}

First Time Diagnosis of Systemic Lupus erythematosus in the 15th Week of Pregnancy with Twins

$\nabla$

In the past it has not been advised to get pregnant when suffering from systemic lupus erythematosus. Fortunately, pregnancy became more usual and manageable among women with systemic lupus erythematosus in the recent years. However, a great number of complications are still bound to occur. Especially in relation with additional renal affection or presence of an antiphospholipid syndrome, a number of complications such as reactivation of systemic lupus erythematodes, thrombosis, miscarriage, lupus erythematodes of the newborn, pregnancy-induced hypertension, pulmonal hypertension and toxicity of medicaments have to be taken care for. Our paper presents the case of the primary diagnosis of a systemic lupus erythematosus affecting a woman in her 15th week of pregnancy with twins. We take this as an opportunity to inform about recent advises concerning such risk pregnancies.

\section{Literatur}

1 Ruiz-Irastorza G, Khamashata MA. Systemischer Lupus erythematodes und Antiphospholipidantikörpersyndrom in der Schwangerschaft. Z für Rheumatologie 2006; Mai 65: 192 - 199

2 Systemischer Lupus erythematodes: www.gesundheitpro.de/ Systemischer-Lupus-Erythematodes-Autoimmunerkrankungen

3 Fischer-Betz R. Rheumatologie. Schwangerschaft und systemischer Lupus erythematodes: leox.dyndns.org: 8080/LupusWebauftritt/jsp/lupus_schwangerschaft_infos. faces; Heinrich-Heine-Universität Düsseldorf

4 Zwahr Ch, Rätz KH, Brietzke W. Letaler Ausgang bei Lupus erythematodes visceralis und Gravidität. Zbl Gynäkologie 1983; 105: 300-307

5 Williams WW, Ecker JL et al. A 29-year-old pregnant woman with nephrotic syndrom and hypertension. New England Journal Of Medicine 2005 Dec ; 353: 2590-2600

6 Braun-Falco O, Plewig G et al. Dermatologie und Venerologie. 5. Auflage. Heidelberg: Springer Verlag,: $696-705$

7 Khamashta MA, Ruiz-Irastorza G et al. Systemic lupus erythematodes flares during pregnancy. Rheum Dis Clin North Am 1997; 23: 15- 30

8 Duckitt $K$, Harrington D. Risk factors of preeclampsia at antenatal booking: systematic review of controlled studies. Br Med J 2005; 330: 565

9 Saaleb S, Copel J et al. Comparison of treatment with fluorinated glucocorticoids to the natural history of autoantibody-associated congenital heart block. Arthritis Rheum 1999; 42: 2335-2345

10 Kuhn A, Ruzicka T. Classification of cutaneous lupus erythematosus. In: Kuhn A, Lehmann P, Ruzicka T (eds).Cutaneous lupus erythematosus. Berlin Heidelberg New York Tokio: Springer,: 53-58

11 Kuhn A, Gensch K, Ständer S, Bonsmann G. Kutaner Lupus erythematodes, Teil 1: Klinik und Klassifikation. Hautarzt 2006; 57: 251 - 268

12 Kuhn A, Sontheimer R, Ruzicka T. Clinical manifestations of cutaneous lupus erythematosus. In: Kuhn A, Lehmann P, Ruzicka T (eds). Cutaneous lupus erythematosus. Berlin Heidelberg New York Tokio: Springer, 2004: 59-92 